\title{
Composição química e toxicidade de óleos essenciais para o pulgão-verde Schizaphis graminum (Rondani, 1852)
}

\author{
Chemical composition and toxicity of essential oils to the green-aphid \\ Schizaphis graminum (Rondani, 1852)
}

\author{
Rafaela Karin Lima1*, Maria das Graças Cardoso², Jair Campos Moraes ${ }^{3}$, Stephan Malfitano Carvalho 4 , \\ Bruno Almeida Melo $^{3}$, Sara Silveira Vieira ${ }^{2}$
}

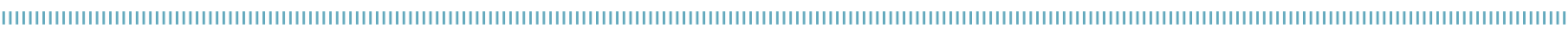

\begin{abstract}
RESUMO: Este trabalho teve como objetivo identificar e quantificar os constituintes dos óleos essenciais de Illicum verum, Ageratum conyzoides, Piper hispidinervum e Ocotea odorifera, bem como avaliar a toxicidade para o pulgão-verde Schizaphis graminum. A qualificação dos constituintes foi realizada por meio de um cromatógrafo gasoso + espectrômetro de massas, e a quantificação, por um cromatógrafo gasoso + detector de ionização de chama, ambos com uma coluna DB5. O método de hidrodestilação promoveu um rendimento (p/p) de 3,81\% para I. verum, $0,46 \%$ para A. conyzoides, $2,85 \%$ para $P$. hispidinervum e $0,68 \%$ para $O$. odorifera. Já os componentes majoritários foram: precoceno $(87,0 \%)$ e (E)-cariofileno (7,1\%) para A. conyzoides; (E)-anetol (90,4\%), limoneno (2,6\%) e metil-chavicol $(1,3 \%)$ para I. verum; metil-eugenol $(81,2 \%)$ e safrol $(10,6 \%)$ para Ocotea odorifera; e safrol $(82,5 \%)$ e $\alpha$-terpinoleno $(13,4 \%)$ para $P$. hispidinervum. Pelos testes de toxicidade aguda (24 horas) com folhas de sorgo ou papel-filtro contaminados, verificou-se que o óleo de $A$. conyzoides foi o mais tóxico para o pulgão, com $\mathrm{CL}_{50}$ de 7,13 e 7,08 $\mu \mathrm{L}$ óleo/ $\mathrm{cm}^{2}$ respectivamente, seguido por O. odorifera com $\mathrm{CL}_{50}$ de 11,80 e $103,00 \mu \mathrm{L}$ óleo/ $\mathrm{cm}^{2}$ respectivamente; I. verum de $51,80 \mu \mathrm{L}$ óleo/ $\mathrm{cm}^{2}$ em ambos os substratos; e o menos tóxico foi o óleo essencial de P. hispidinervum, com $\mathrm{CL}_{50}$ de 62,50 e 143,00 $\mu \mathrm{L}$ óleo/ $\mathrm{cm}^{2}$, respectivamente. Dessa maneira, sugere-se que o uso dos óleos essenciais pode representar uma nova ferramenta em programas de manejo integrado de pragas.
\end{abstract}

PALAVRAS-CHAVE: Illicum verum; Ageratum conyzoides; Piper hispidinervum; Ocotea odorifera; compostos aromáticos; atividade inseticida; Aphididae.

\begin{abstract}
The aim of this study was to assess the chemical composition of essential oils of Illicum verum, Ageratum conyzoides, Piper hispidinervum and Ocotea odorifera, as well as their toxicity to the green-aphid Schizaphis graminum. Compound identification was carried out with gas chromatography + mass spectrometry, and quantification with gas chromatography + flame ionization detector, both with DB5 column. The hydrodistillation process promoted oil yield of $3.81 \%$ for $I$. verum; $0.46 \%$ for $A$. conyzoides; $2.85 \%$ for $P$. hispidinervum; and $0.68 \%$ for $O$. odorifera. The major components from the oils of $A$. conyzoides was precocene $(87.0 \%)$ and (E)-caryophyllene (7.1\%); (E)-anethol (90.4\%), limonene (2.6\%) and methyl-chavicol (1.3\%) for I. verum; methyleugenol $(81.2 \%)$ and safrole (10.6\%) for O. odorifera; and safrole (82.5\%) and $\alpha$-terpinolene $(13.4 \%)$ for $P$. hispidinervum. The acute toxicity test ( 24 hours) performed by contact in sorghum leaves or contaminated filter-paper showed that the higher toxicity occur with essential oils of $A$. conyzoides, with $\mathrm{LC}_{50}$ of 7.01 and $7.01 \mu \mathrm{g}$ oil $/ \mathrm{cm}^{2}$, respectively; followed by $O$. odorifera, with $\mathrm{LC}_{50}$ of 17.10 and $66.70 \mu \mathrm{g} \mathrm{oil} / \mathrm{cm}^{2}$, respectively; I. verum, with $\mathrm{LC}_{50}$ of 66.40 and $65.40 \mu \mathrm{g}$ oil $/ \mathrm{cm}^{2}$, respectively; and low toxicity was observed for the P. hispidinervum essential oil, with $\mathrm{LC}_{50}$ of 64.00 and $143.00 \mathrm{oil} / \mathrm{cm}^{2}$, respectively. With the results obtained in this research, we can state that the use of these essential oils can be a new tool in integrated pest management.
\end{abstract}

KEYWORDS: Illicum verum; Ageratum conyzoides; Piper hispidinervum; Ocotea odorifera; aromatic compounds; insecticidal activity; Aphididae. 


\section{INTRODUÇÃO}

O pulgão Schizaphis graminum (Rondoni, 1852) (Hemiptera: Aphididae) é um importante inseto-praga das culturas de trigo e sorgo, presente nas Américas do Norte e Sul, Europa, África e no meio-oeste da Ásia (Blackman; Eastop, 2000). Seu ataque ocorre em todos os estádios fenológicos das plantas, mas preferencialmente em novas brotaçóes, acarretando danos pela sucçáo da seiva, transmissão de vírus, injeção de toxinas e redução do estande (SAlvadori; Tonet, 2001). Em detrimento aos métodos convencionais de controle, empregando inseticidas sintéticos de amplo espectro de ação, o uso de produtos naturais, tais como os óleos essenciais, destaca-se por apresentar bons índices de controle. Além disso, possibilitam o uso integrado em programas de Manejo Integrado de Pragas, tornando-se uma alternativa ecologicamente correta para o controle de insetos-praga (Regnault-Roger, 1997; Coitinho et al., 2010).

Nesse sentido, Mendonça et al. (2005) verificaram que extratos e óleos essenciais de plantas brasileiras possuem ação inseticida contra larvas de Aedes aegypti (Linnaeus, 1762), fazendo desses compostos uma importante fonte de estudo no controle desse vetor. Estrela et al. (2006) estudaram os óleos essenciais de duas espécies do gênero Piper e concluíram que ambos são tóxicos para Sitophilus zeamais Mots., 1855. Já FAzOLin et al. (2007) concluíram que os óleos essenciais de duas piperáceas e uma bignoniácea foram tóxicos ao Tenebrio molitor L., 1758; bem como Lima et al. (2009), avaliando o óleo essencial de Piper hispidinervum C. DC. (Piperaceae) sobre Spodoptera frugiperda (J. E. SмIтH, 1797). Com relação ao controle de afídeos, trabalhos recentemente publicados comprovaram que os óleos essenciais sáo uma alternativa eficiente para o controle dessas pragas. Por exemplo, os óleos essenciais de citronela, mentrasto, anis-estrelado, pimenta-longa, alfazema, entre outros, foram eficazes no controle dos pulgões (Hymenoptera: Aphididae) Myzus persicae Sulzer, 1776, Macrosiphum euphorbiae Thomas, 1878, Aphis gossypii Glover, 1877, Brevicoryne brassicae Linnaeus, 1758 e Hyadaphis foeniculi Passerini, 1860 (Pavela, 2006; Abramson et al., 2006; Soares et al., 2011; Soares et al., 2012; Andrade et al., 2013; Pinheiro et al., 2013).

De maneira geral, a atividade tóxica dos óleos essenciais sobre diversos micro-organismos e artrópodes-praga pode estar relacionada ao conjunto de substâncias em sua composição, e não somente a cada um dos compostos majoritários (VARDar-Unlü et al., 2003). Portanto, um dos fatores mais relevantes no estudo de sua aplicabilidade é sua composição química, a qual pode variar em uma mesma planta devido a fatores ligados à biologia (genética, nutrição e fase de desenvolvimento), além daqueles edafoclimáticos (local, condiçôes climáticas e tipo de solo) (Lima et al., 2003).

As espécies pimenta-longa $P$. hispidinervum e canela-sassafrás Ocotea odorifera Vellozo Rohwer (Lauraceae) são largamente encontradas na região amazônica, apresentando em seu óleo essencial o safrol como principal constituinte. Este fenilpropano se destaca devido às propriedades antimicrobiana e inseticida, sendo utilizado como fonte para a síntese de vários medicamentos, inclusive de alguns piretroides. O mentrasto, Ageratum conyzoides Linnaeus (Asteraceae), é uma planta conhecida no Brasil e usada na medicina popular por seu potencial fitoterápico (analgésico e cicatrizante), sendo que seu óleo é composto principalmente por precocenos (I e II) (LoREnZI; MATOs, 2002). $\mathrm{O}$ anis-estrelado, Illicium verum Hook. F. (Schisandraceae), é muito utilizado como condimento e cosmético, podendo conter como constituinte majoritário o (E)-anetol, o qual possui ação fungicida contra dermatófitos (Kosalec et al., 2005).

Nesse contexto, objetivou-se identificar e quantificar os constituintes dos óleos essenciais de I. verum, A. conyzoides, $P$. hispidinervum e $O$. odorifera, bem como avaliar a toxicidade sobre o pulgão-verde S. graminum em condições de laboratório.

\section{MATERIAL E MÉTODOS}

\section{Obtenção dos óleos essenciais}

O óleo essencial de I. verum foi obtido a partir de $100 \mathrm{~g}$ de frutos secos adquiridos no comércio local de Lavras (MG), e aqueles de $A$. conyzoides, $P$. hispidinervum e $O$. odorifera, a partir de $300 \mathrm{~g}$ de folhas frescas coletadas no horto de plantas medicinais da Universidade Federal de Lavras (UFLA) $\left(21^{\circ} 14^{\prime} \mathrm{S} ; 45^{\circ} 00^{\prime} \mathrm{W}\right.$, altitude de $919 \mathrm{~m}$ e temperatura média anual de $26^{\circ} \mathrm{C}$ ) durante o período da manhã e entre os meses de abril e maio de 2007. Cada espécime foi identificado e catalogado no herbário ESAL do Departamento de Biologia da UFLA (A. conyzoides reg. 12.726; P. hispidinervum reg. 23.013; O. odorifera reg. 22.215).

O processo de obtenção dos óleos essenciais foi o de hidrodestilaçáo por meio de um aparelho de Clevenger modificado, com duração de 2,5 horas, pelo qual o hidrolato obtido foi centrifugado a $965 \mathrm{~g}$ a $25^{\circ} \mathrm{C}$, durante 5 minutos, para promover a separaçáo entre as fases aquosa e oleosa, sendo esta última coletada e armazenada em frasco de vidro âmbar a $4^{\circ} \mathrm{C}$ até a realização das análises posteriores (Lima et al., 2009).

\section{Análise qualitativa dos óleos essenciais}

A identificaçáo dos componentes foi realizada por meio de cromatografia gasosa utilizando um equipamento Shimadzu (GC-17A) acoplado a um espectrômetro de massas com detector seletivo (QP 5000). Foi empregada uma coluna do tipo capilar de sílica fundida e fase ligada (DB5, $30 \mathrm{~m} \mathrm{x} \mathrm{0,25} \mathrm{mm),}$ sendo a fase móvel o gás hélio $(1 \mathrm{~mL} / \mathrm{min}$.), com temperaturas de $220^{\circ} \mathrm{C}$ no injetor e $240^{\circ} \mathrm{C}$ no detector. A temperatura 
do forno foi de 40 a $240^{\circ} \mathrm{C}$, com acréscimo de $3^{\circ} \mathrm{C} / \mathrm{min}$; a pressão inicial na coluna foi de $100,2 \mathrm{KPa}$; taxa de split 1:10 e volume injetado de $1 \mu \mathrm{L}$ (soluçóes a $1 \%$ (v/v) em diclorometano). Nas mesmas condiçóes da amostra, foi injetada uma série de padrôes de hidrocarbonetos $\left(\mathrm{C}_{9} \mathrm{H}_{20}\right.$ à $\left.\mathrm{C}_{26} \mathrm{H}_{54}\right)$, gerando a regressão $f(x)=25.294 \mathrm{TR}+610.630$, na qual TR é o tempo de retençáo de cada composto. Dessa maneira, foram comparados os espectros de massa de cada composto com o banco de dados da biblioteca Wiley 229, como também pelo índice Kovat's tabelado (Adams, 2007).

\section{Análise quantitativa dos óleos essenciais}

A quantificação das substâncias foi realizada a partir da área dos picos obtidos nos cromatogramas, expressa em percentagem da área total, sendo realizada em triplicata, obtendo-se assim a média e o desvio padrão. Para isso, utilizou-se um cromatógrafo Shimadzu (GC-17A) equipado com detector de ionização de chama de hidrogênio e coluna capilar de sílica fundida e fase ligada (DB5, $30 \mathrm{~m}$ x 0,25 mm). O gás de arraste foi o nitrogênio $(2,2 \mathrm{~mL} / \mathrm{min}$.); taxa split de 1:10 e volume de amostra injetado de $1 \mu \mathrm{L}$ (soluçóes a 1\% (v/v) em diclorometano). A temperatura inicial da coluna foi de $40^{\circ} \mathrm{C}$ com acréscimo de $3^{\circ} \mathrm{C} / \mathrm{min}$., até $240^{\circ} \mathrm{C}$. As temperaturas do injetor e do detector foram fixadas em $220^{\circ} \mathrm{C}$ e $240^{\circ} \mathrm{C}$, respectivamente, com a pressão da coluna de $115 \mathrm{KPa}$.

\section{Avaliação toxicológica dos óleos essenciais sobre o pulgão-verde}

Pulgóes adultos ápteros com idade média de 2 a 3 dias foram obtidos em uma criação de manutenção do Departamento de Entomologia da UFLA, usando folhas de Sorghum bicolor (L.) Moench (Poacea) cultivar BR-85, temperatura de $26 \pm 2^{\circ} \mathrm{C}$, umidade relativa de $70 \pm 10 \%$ e fotofase de 14 horas.

Para a determinação dos valores de concentração letal $\left(\mathrm{CL}_{10} ; \mathrm{CL}_{50}\right.$ e $\left.\mathrm{CL}_{90}\right)$, diversas concentraçóes dos óleos essenciais foram preparadas diluindo-as em acetona, obtendo gamas de concentraçáo (v/v) 0,025 a 3,0\% para $A$. conyzoides; 0,3 a $2,0 \%$, para I. verum; 1,0 a 2,5\%, para P. hispidinervum; e 0,2 a 2,6\%, para $O$. odorifera. No caso dos tratamentos controle, foi utilizado somente o solvente acetona. Os ensaios de toxicidade aguda foram realizados em delineamento inteiramente casualizado e esquema fatorial ( 4 óleos $\mathrm{x} 2$ substratos $\mathrm{x} 9$ concentraçóes), sendo que cada parcela amostral foi composta por uma placa de Petri com dez pulgóes adultos ápteros e repetidos seis vezes, totalizando 60 indivíduos por concentração.

Em um primeiro experimento, discos foliares de sorgo foram utilizados como substrato para a contaminação com os óleos essenciais, bem como para a alimentação dos pulgóes. Seçóes circulares de 19,6 $\mathrm{cm}^{2}$ foram higienizadas com hipoclorito de sódio a 1\%, enxaguadas com água destilada, acondicionadas e fixadas em placa de Petri (5 cm de diâmetro) por uma solução de água:ágar a 2\% e fechadas por filme de PVC. No segundo experimento, devido ao interesse em verificar somente o efeito tóxico dos óleos essenciais, sem a alimentaçáo dos pulgóes, contaminaram-se seçôes circulares de $19,6 \mathrm{~cm}^{2}$ de papel-filtro, acondicionando-as no fundo das placas de Petri, e fechando-as com filme de PVC. Em ambos os experimentos, utilizando folha de sorgo ou papel-filtro, as superfícies foram contaminadas uniformemente pela distribuição de $4 \mathrm{~mL}$ de uma das soluçôes de acetona:óleo essencial, a qual foi realizada com auxílio de uma micropipeta automática (GuTIÉRREZ et al., 1997). Devido à impossibilidade de manutenção dos pulgóes durante um período de 48 horas sem alimentaçáo, o ensaio de contaminaçấo em papel-filtro teve duração máxima de 24 horas. No ensaio em que as folhas de sorgo foram contaminadas com os óleos essenciais, as avaliaçôes ocorreram 24 e 48 horas após a liberação dos pulgóes. Durante as avaliaçôes, foram também considerados como pulgóes mortos aqueles que não respondiam a estímulos.

Os dados de mortalidade foram submetidos à análise estatística do tipo dose-resposta, empregando-se modelos logísticos do pacote DRC (Analysis of Dose-Response Curves), compilado pelo software $\mathrm{R}^{\prime}$ (2010). Com a escolha dos melhores modelos, foram entáo estimados os valores das concentraçôes letais $\left(\mathrm{CL}_{10} ; \mathrm{CL}_{50}\right.$ e $\left.\mathrm{CL}_{90}\right)$ com os respectivos intervalos de confiança $(95 \%)$.

\section{RESULTADOS E DISCUSSÃO}

\section{Identificação e quantificação dos constituintes dos óleos essenciais}

Pelo processo de hidrodestilação empregado, obteve-se um rendimento (p/p) de óleo essencial na ordem de 3,81\% para I. verum; $2,85 \%$ para P. hispidinervum; $0,68 \%$ para $O$. odorifera; e $0,46 \%$ para $A$. conyzoides. De maneira geral, observa-se que os rendimentos obtidos estáo de acordo com aqueles apresentados por Rodrigues et al. (2003) para I. verum $(3,31 \% \mathrm{p} / \mathrm{p})$; por CAstro et al. (2004), para o óleo essencial de $A$. conyzoides, os quais observaram uma variaçáo de 0,49 a $0,70 \%(\mathrm{p} / \mathrm{p})$; e por FAzolin et al. (2007), que verificaram um rendimento de 3 a $3,5 \%(\mathrm{p} / \mathrm{p})$ para o óleo de P. hispidinervum. Somente para $O$. odorifera, o rendimento encontrado neste estudo foi inferior ao relatado por Castellani et al. (2006), já que, segundo esses autores, a variação está ligada em função da coleta durante o período de outono.

O óleo essencial de $A$. conyzoides apresentou o precoceno $(87,00 \%)$ como seu composto majoritário, seguido do (E)-cariofileno $(7,10 \%)$, e, em menores concentraçóes, os sesquiterpenos $\beta$-cubebeno, $\alpha$-humuleno, germancreno-D e $\gamma$-cadineno (Tabela 1), resultados que são concordantes com os obtidos por Kong et al. (1999). Segundo Castroet al. (2004), 
existe uma grande variação na composição dos óleos essenciais de $A$. conyzoides em função do local de coleta das amostras. Contudo, os resultados aqui obtidos corroboram aqueles apresentados por esses autores. No que se refere ao óleo essencial da espécie $I$. verum, encontrou-se na maioria das vezes o composto (E)-anetol $(90,40 \%)$, em menores quantidades, o limoneno $(2,60 \%)$ e metil-chavicol (1,30\%), além de pequenas concentraçôes do $\alpha$-pineno, linalol e 4-terpineol. Esses dados estáo de acordo com aqueles apresentados por RoDRIGUES et al. (2003), que encontraram maioria de (E)-anetol (90,00\%), além de outros compostos, como o seu isômero, $(Z)$-anetol, e os fenilpropanoides metil-chavicol e anisaldeído. Esses autores destacam que pequenas diferenças podem ocorrer entre alguns compostos, provavelmente relacionadas aos processos de extração, quantificação/identificação, além de alterações ligadas à origem do material vegetal, local de coleta, clima e fertilidade do solo.

No caso do óleo de $P$. hispidinervum, verificou-se em sua constituição o safrol $(82,50 \%)$ e o $\alpha$-terpinoleno $(13,40 \%)$, em maiores proporçóes, e em menores concentraçóes os monoterpenos $\alpha$-pineno e $\delta$-3-careno (Tabela 1 ). Esses resultados divergem dos encontrados por FAzOLIN et al. (2007) que, usando plantas originadas do estado do Acre, verificaram que a porcentagem de safrol contida em plantas de P. hispedinervum era superior a 90,00\%. Novamente, esta diferença pode estar associada a diversos fatores bióticos e abióticos aos quais determinada planta pode estar exposta, tais como insolaçáo, tipo de solo, umidade relativa, presença de pragas e doenças. Esses fatores podem induzir diferentes mecanismos bioquímicos envolvidos na síntese do metabolismo secundário e, consequentemente, nas composiçôes dos óleos essenciais (Simốes; Sptizer, 2004). Para $O$. odorifera foi encontrado um alto teor dos fenilpropanoides, como o metil-eugenol $(81,20 \%)$, seguido do safrol (10,60\%), e, em pequenas proporçóes, 1,8-cineol e cânfora. Algumas pesquisas observaram que os teores de safrol e de metil-eugenol são variáveis, novamente dependendo da região de ocorrência dessa espécie vegetal (Rızzini; Mors, 1995). Ressalta-se que a variação no rendimento do óleo essencial de $O$. odorifera está diretamente ligada ao período de coleta e à fonte vegetal usada (folha, galhos, cascas), conforme descrito por Castellani et al. (2006).

\section{Efeitos toxicológicos dos óleos essenciais}

Entre os quatro óleos essenciais avaliados, verificou-se que aquele originado de $A$. conyzoides foi o mais tóxico, apresentando

Tabela 1. Composição química, concentração (\% \pm E.P.), índices de Kovat's calculado (IK $)$ (baseados nos tempos de retenção de uma mistura para uma série de n-alcanos) e índices de Kovat's tabelado (IK) (Adams, 2007) dos óleos essenciais de Ageratum conyzoides, Illicum verum, Piper hispidinervum e Ocotea odorifera.

\begin{tabular}{|c|c|c|c|c|}
\hline Espécie vegetal & Composto & Concentração & $\mathrm{IK}_{\mathrm{c}}$ & $\mathrm{IK}_{\mathrm{t}}$ \\
\hline \multirow{6}{*}{ Ageratum conyzoides } & precoceno & $87,02 \pm 0,14$ & 1472 & 1469 \\
\hline & (E)-cariofileno & $7,08 \pm 0,13$ & 1410 & 1404 \\
\hline & $\alpha$-humuleno & $1,18 \pm 0,02$ & 1459 & 1454 \\
\hline & $\gamma$-cadineno & $1,18 \pm 0,06$ & 1517 & 1513 \\
\hline & $\beta$-cubeno & $0,69 \pm 0,00$ & 1394 & 1390 \\
\hline & germancreno-D & $0,52 \pm 0,01$ & 1486 & 1480 \\
\hline \multirow{5}{*}{ Illicum verum } & (E)-anetol & $90,41 \pm 0,29$ & 1286 & 1283 \\
\hline & limoneno & $2,65 \pm 0,17$ & 1031 & 1031 \\
\hline & metil-chavicol & $1,26 \pm 0,01$ & 1201 & 1195 \\
\hline & linalol & $1,07 \pm 0,02$ & 1110 & 1098 \\
\hline & $\alpha$-pineno & $0,35 \pm 0,03$ & 933 & 939 \\
\hline \multirow{4}{*}{ Piper hispidinervum } & safrol & $82,40 \pm 0,80$ & 1290 & 1285 \\
\hline & $\alpha$-terpinoleno & $13,38 \pm 0,65$ & 1088 & 1088 \\
\hline & $\delta$-3-careno & $1,30 \pm 0,07$ & 1010 & 1011 \\
\hline & $\alpha$-pineno & $0,68 \pm 0,05$ & 933 & 939 \\
\hline \multirow{4}{*}{ Ocotea odorifera } & metil-eugenol & $81,20 \pm 1,70$ & 1407 & 1401 \\
\hline & safrol & $10,60 \pm 0,56$ & 1290 & 1285 \\
\hline & cânfora & $5,87 \pm 0,77$ & 1145 & 1143 \\
\hline & 1,8-cineol & $0,64 \pm 0,17$ & 1030 & 1033 \\
\hline
\end{tabular}


valores de $\mathrm{CL}_{50}$ no ensaio com folha de sorgo contaminada de 7,13 e $2,50 \mu \mathrm{L}$ óleo $/ \mathrm{cm}^{2}$, após 24 e 48 horas, respectivamente; e no de papel-filtro, $7,08 \mu \mathrm{L}$ óleo $/ \mathrm{cm}^{2}$, após 24 horas. Os valores de $\mathrm{CL}_{10}$ e $\mathrm{CL}_{90}$ foram de 0,91 e $54,80 \mu \mathrm{L}$ óleo/ $\mathrm{cm}^{2}$ (24 horas) e 0,26 e $26,80 \mu \mathrm{L}$ óleo $/ \mathrm{cm}^{2}$ ( 48 horas) no ensaio com folha de sorgo e de 0,97 e 52,70 óleo/ $\mathrm{cm}^{2}$ (24 horas), quando usando papel-filtro com superfície contaminada (Tabela 2). Verificaram-se que os valores das concentraçôes letais no período de 24 horas nos ensaios com folhas de sorgo e papel-filtro contaminados foram similares, evidenciando a alta toxicidade desse óleo para $S$. graminum.

A alta toxicidade do óleo essencial de $A$. conyzoides verificada neste estudo está de acordo com Regnault-Roger (1997) e Okunade (2002), que afirmaram que este óleo essencial pode se tornar uma excelente alternativa para o uso no controle integrado de pragas. Como observado aqui, Bouda et al. (2001) também concluíram que o óleo essencial de A. conyzoides é tóxico para Sitophilus zeamais (Mots.), principalmente pela presença dos precocenos I e II, apresentando $\mathrm{CL}_{50}$ de $0,09 \%(\mathrm{p} / \mathrm{p})$. Segundo Mendonça et al. (2005), A. aegypti foi altamente suscetível ao óleo essencial de A. conyzoides, com $100 \%$ de mortalidade das larvas após 48 horas e $\mathrm{CL}_{50}$ de $148 \mu \mathrm{g}$ óleo/L água. SAXena et al. (1992) e OKunade (2002) atribuíram a toxicidade desse óleo essencial à presença de precocenos, os quais podem ter ação sobre o hormônio juvenil, acelerando o processo de metamorfose e originando adultos com alteraçóes morfológicas e fisiológicas. Recentemente, SoAres et al. (2011) verificaram que o óleo essencial de $A$. conyzoides aplicado em dosagens superiores a 0,5\%, foi capaz de matar $100 \%$ dos pulgóes M. euphorbiae em roseira, caracterizando o potencial inseticida deste composto e justificando sua utilizaçấo como uma alternativa no controle de insetos-praga.

O óleo essencial de I. verum demonstrou ser tóxico aos pulgóes, porém, em concentraçóes mais elevadas, com $\mathrm{CL}_{50}$ de 51,80 e 40,50 $\mu \mathrm{L}$ óleo/ $\mathrm{cm}^{2}$ (24 e 48 horas) quando foram utilizadas folhas de sorgo, e de 51,80 $\mu$ g óleo/ $\mathrm{cm}^{2}$ (24 horas), com a utilizaçáo de discos de papel-filtro (Tabela 2). Os valores de $\mathrm{CL}_{10}$ e $\mathrm{CL}_{90}$ no ensaio com folha de sorgo foram, respectivamente, 24,60 e 110,00 $\mu \mathrm{L}$ óleo/ $\mathrm{cm}^{2}$ (24 horas) e 14,90 e $109,00 \mu \mathrm{L}$ óleo/ $\mathrm{cm}^{2}$ (48 horas), e com papel-filtro, de 24,60 e 109,00 $\mu \mathrm{L}$ óleo $/ \mathrm{cm}^{2}$ (24 horas) (Tabela 2).

Observa-se que independentemente do tipo da superfície contaminada, elevadas taxas de mortalidade sáo encontradas quando os pulgóes $S$. graminum entram em contato com o óleo essencial de I. verum, caracterizando-o como promissor para o controle desse pulgão. Similarmente, SoARES et al. (2012) também comprovaram que o óleo essencial de

Tabela 2. Concentração letal $\left(\mathrm{CL}_{10} ; \mathrm{CL}_{50}\right.$ e $\mathrm{CL}_{90}-\mu \mathrm{L}$ óleo essencial/ $\left.\mathrm{cm}^{2}\right)$ dos óleos essenciais utilizando os substratos folha de sorgo e papel-filtro, as 24 e 48 horas após a liberação de adultos do pulgão verde Schizaphis graminum.

\begin{tabular}{|c|c|c|c|c|}
\hline \multirow{3}{*}{ Óleo essencial } & & \multicolumn{3}{|c|}{ Parâmetros estimados } \\
\hline & & \multicolumn{2}{|c|}{ Folha de sorgo } & \multirow{2}{*}{$\begin{array}{l}\text { Papel-filtro } \\
24 \text { horas }\end{array}$} \\
\hline & & 24 horas & 48 horas & \\
\hline \multirow{4}{*}{ Ageratum conyzoides } & $\mathrm{CL}_{10(\mathrm{IC} 95 \%)}$ & $0,91_{(0,56-1,53)}$ & $0,26_{(0,10-0,46)}$ & $0,97_{(0,56-1,53)}$ \\
\hline & $\mathrm{CL}_{50(\text { IC95\%) }}$ & $7,13_{(5,40-9,38)}$ & $2,50_{(1,78-3,52)}$ & $7,08_{(5,40-9,27)}$ \\
\hline & $\mathrm{CL}_{90 \text { (IC95\%) }}$ & $54,80_{(35,80-83,80)}$ & $26,8_{(18,00-39,70)}$ & $52,70_{(35,10-79,40)}$ \\
\hline & $\mathrm{GL}-\chi^{2}-\mathrm{n}$ & $36-41,98-400$ & $50-56,59-500$ & $38-43,37-400$ \\
\hline \multirow{4}{*}{ Illicum verum } & $\mathrm{CL}_{10(\mathrm{IC} 95 \%)}$ & $24,60_{(21,00-28,70)}$ & $14,90_{(11,80-18,80)}$ & $24,60_{(21,00-28,70)}$ \\
\hline & $\mathrm{CL}_{50(\text { IC95\%) }}$ & $51,80_{(48,20-55,70)}$ & $40,50_{(36,60-44,70)}$ & $51,80_{(48,20-55,70)}$ \\
\hline & $\mathrm{CL}_{90(1 \mathrm{C} 95 \%)}$ & $110,00_{(93,70-130,00)}$ & $109,00_{(95,60-125,40)}$ & $109,00_{(95,60-125,00)}$ \\
\hline & $\mathrm{GL}-\chi^{2}-\mathrm{n}$ & $55-59,00-510$ & $58-50,92-510$ & $55-59,00-510$ \\
\hline \multirow{4}{*}{ Piper hispidinervum } & $\mathrm{CL}_{10(\mathrm{IC} 95 \%)}$ & $41,40_{(28,10-61,00)}$ & $29,20_{(21,80-39,20)}$ & $121,00_{(109,00-133,00)}$ \\
\hline & $\mathrm{CL}_{50(\text { IC95\%) }}$ & $62,50_{(51,50-75,80)}$ & $55,10_{(48,30-62,90)}$ & $143,00_{(136,00-151,00)}$ \\
\hline & $\mathrm{CL}_{90(\text { (IC95\%) }}$ & $104,00_{(94,10-115,00)}$ & $94,30_{(81,30-109,00)}$ & $170,00_{(161,00-181,00)}$ \\
\hline & $\mathrm{GL}-\chi^{2}-\mathrm{n}$ & $53-58,44-570$ & $46-38,02-450$ & $54-28,64-570$ \\
\hline \multirow{4}{*}{ Ocotea odorifera } & $\mathrm{CL}_{10(\mathrm{IC} 95 \%)}$ & $3,26_{(1,78-5,91)}$ & $3,16_{(1,68-5,86)}$ & $83,50_{(70,90-98,30)}$ \\
\hline & $\mathrm{CL}_{50(\text { IC95\%) }}$ & $11,80_{(8,61-16,20)}$ & $11,70_{(8,46-16,20)}$ & $103,00(95,20-111,00)$ \\
\hline & $\mathrm{CL}_{90(\mathrm{IC95 \% )}}$ & $43,20_{(34,30-54,30)}$ & $42,50_{(34,00-53,10)}$ & $127,00_{(118,00-136,00)}$ \\
\hline & $\mathrm{GL}-\chi^{2}-\mathrm{n}$ & $46-34,56-450$ & $43-30,45-420$ & $48-32,19-450$ \\
\hline
\end{tabular}

IC95\%: intervalo de confiança a 95\%; GL: grau de liberdade; $\chi^{2}$ : qui-quadrado do modelo; n: número total de indivíduos testados. 
I. verum apresenta ação inseticida e foi capaz de controlar os pulgóes $M$. euphorbiae em roseira. Esses resultados são concordantes com aqueles obtidos por Ho et al. (1997), os quais verificaram que ovos, larvas e adultos de Tribolium castaneum (Herbst) e $S$. zeamais, são suscetíveis quando estão em contato com superfícies tratadas com derivados de I. verum. Chang; AHN (2001) concluíram que o fenilpropeno (E)-anetol, componente majoritário do óleo essencial de I. verum, é tóxico à Blattella germanica (L.) igualando-se a inseticidas sintéticos.

Semelhante ao óleo essencial de $A$. conyzoides (Tabela 2), verificou-se a toxicidade do óleo essencial de $O$. odorifera aos pulgóes, com valores de $\mathrm{CL}_{50}$ de 11,80 e 11,70 $\mu \mathrm{L}$ óleo/ $\mathrm{cm}^{2}$ (24 horas e 48 horas, respectivamente), no ensaio com folhas de sorgo, e de 103,00 $\mu \mathrm{L}$ óleo/ $\mathrm{cm}^{2}$ (24 horas) com o papel-filtro. Os valores de $\mathrm{CL}_{10}$ foram de 3,26 e 3,16 $\mu \mathrm{L}$ óleo $/ \mathrm{cm}^{2}$ (24 e 48 horas em folha de sorgo) e $83,50 \mu \mathrm{L}$ óleo/ $\mathrm{cm}^{2}$ (24 horas em papel-filtro), e os valores de $\mathrm{CL}_{90}$ foram de 43,20 e 42,50 $\mu \mathrm{L}$ óleo $/ \mathrm{cm}^{2}$ (24 e 48 horas em folha de sorgo) e 127,00 $\mu \mathrm{L}$ óleo $/ \mathrm{cm}^{2}$ (24 horas em papel-filtro). Observa-se que no ensaio com papel-filtro as concentraçôes letais foram superiores, demonstrando serem menos tóxicas nessa forma de contaminação em relação à contaminação em folhas de sorgo. Provavelmente, a diferença encontrada entre os valores de CL nos dois substratos está relacionada à maior exposiçáo durante o caminhamento e/ou pelo ato de introdução do aparelho bucal na folha para alimentação.

Embora não se tenha conhecimento sobre estudos toxicológicos utilizando pulgóes, resultados semelhantes aos da presente pesquisa foram obtidos por NGOH et al. (1998) com os fenilpropanóides metil-eugenol e safrol, substâncias essas encontradas em abundância no óleo essencial de $O$. odorifera. Durante os ensaios de intoxicação por contato, esses autores concluíram que metil-eugenol e safrol apresentam efeito neurotóxico e provocam a morte de Periplaneta americana (L.). No caso específico de metil-eugenol, este composto apresentou efeito knockdown, de maneira semelhante aos inseticidas do grupo dos piretroides, sugerindo que o mesmo possa estar interagindo, direta ou indiretamente, com o processo de transmissão axônica. No caso do metil-eugenol, HuANG et al. (2002) também verificaram que esse composto possui ação tóxica sobre $S$. zeamais e $T$. castaneum.

Dentre todos, o óleo essencial de $P$. hispidinervum foi o menos tóxico aos pulgóes, sendo a $\mathrm{CL}_{50}$ no ensaio com folhas de sorgo contaminadas de 62,50 e $55,10 \mu \mathrm{L}$ óleo $/ \mathrm{cm}^{2}$ (24 e 48 horas), e de $143,00 \mu \mathrm{L}$ óleo/ $\mathrm{cm}^{2}$ em papel-filtro (24 horas). As demais concentraçóes letais foram: $\mathrm{CL}_{10}$ de 41,40 e $29,20 \mu \mathrm{L}$ óleo/ $\mathrm{cm}^{2}$ (24 e 48 horas; folha de sorgo) e $121,00 \mu \mathrm{L}$ óleo/ $\mathrm{cm}^{2}$ (24 horas; papel-filtro). $\mathrm{As}^{\mathrm{CL}_{90}}$ foram de 104,00 e 94,30 $\mu \mathrm{L}$ óleo/ $\mathrm{cm}^{2}$ (24 e 48 horas; folha de sorgo) e $170,00 \mu \mathrm{L}$ óleo/ $\mathrm{cm}^{2}$ (24 horas; papel-filtro) (Tabela 2).
Comparando-se os valores de concentração letal do óleo de $P$. hispidinervum entre as duas superfícies contaminadas (24 horas), observou-se que no ensaio com folhas de sorgo os valores $\left(\mathrm{CL}_{50}\right)$ são substancialmente menores, em média 2,28 vezes em relação ao papel-filtro. Como observado com o óleo essencial de $O$. odorifera, sugere-se que a diferença entre os valores esteja relacionada à maior exposição dos pulgóes ao óleo essencial durante o caminhamento e/ou alimentação, como também pelo modo de ação dos compostos. Considerando o aspecto comportamental e o desenvolvimento de A. gossypii, Andrade et al. (2013) verificaram que o óleo essencial de $P$. hispidinervum não afetou nem a repelência nem a quantidade de ninfas deste afídeo. Entretanto, conforme também observado em nosso estudo, SoAREs et al. (2012) observaram que este óleo essencial foi tóxico para os pulgóes $M$. euphorbiae, justificando que a ação inseticida está diretamente correlacionada com a presença do (E)-anetol. Toxicidade semelhante à observada neste trabalho foi obtida por $\mathrm{NGOH}$ et al. (1998), após exporem $P$. americana ao contato com papel-filtro contaminado com safrol. Eles concluíram que esse composto majoritário do óleo essencial de $P$. hispidinervum é neurotóxico e induz ao knockdown. Em outro estudo, Huang et al. (1999) concluíram que o safrol obtido em óleo essencial de P. hispidinervum possui potencial tóxico por contato e fumigação frente a $S$. zeamais e $T$. castaneum, além de apresentar efeito de deterrência para adultos de $S$. zeamais quando em concentraçóes superiores a $8,11 \mathrm{mg}$ safrol/g de alimento.

\section{CONCLUSÃO}

Os componentes majoritários encontrados foram o precoceno e o (E)-cariofileno para A. conyzoides; metil-eugenol e safrol, para O. odorifera; (E)-anetol para I. verum; safrol e $\alpha$-terpinoleno, para P. hispidinervum. Pelos estudos toxicológicos, observaram-se que ambos os óleos foram tóxicos para $S$. graminum, sendo a ordem de toxicidade: A. conyzoides, O. odorifera, I. verum e $P$. hispidinervum. Portanto, conclui-se que o uso de óleos essenciais pode constituir uma alternativa para o controle desse afídeo.

\section{AGRADECIMENTOS}

Gostaríamos de agradecer à Coordenação de Aperfeiçoamento de Pessoal de Nível Superior (CAPES), ao Conselho Nacional de Desenvolvimento Científico e Tecnológico (CNPq) e à Fundação de Amparo à Pesquisa do Estado de Minas Gerais (FAPEMIG) pelos apoios financeiros. 


\section{REFERÊNCIAS}

ABRAMSON, C.I.; WANDERLEY, P.A.; WANDERLEY, M.J.A.; MINÁ, A.J.S.; SOUZA, O.B. Effect of essential oil from citronella and alfazema on fennel aphids Hyadaphis foeniculi Passerini (Hemiptera: Aphididae) and its predator Cycloneda sanguinea L. (Coleoptera: Coccinelidae). American Journal of Environmental Sciences, v.3, n. 1, p.9-10, 2006.

ADAMS, R.P. Identification of essential oil components by gas chromatography/mass spectroscopy. Ilinois: Allured Publishing Corporation, 2007. 804p.

ANDRADE, L.H.; OLIVEIRA, J.V.; LIMA, I.M.M.; SANTANA, M.F.; BREDA, M.O. Efeito repelente de azadiractina e óleos essenciais sobre Apis gossypii Glover (Hemiptera: Aphididae) em algodoeiro. Revista Ciência Agronômica, v.44, n.3, p.628-634, 2013.

BLACKMAN, R.L.; EASTOP, V.F. Aphis on the world's crops: An identification and information guide. London: John Wiley \& Sons, 2000. 466p.

BOUDA, H.; TAPONDJOU, L.A.; FONTEM, D.A.; GUMEDZOE, M.Y.D. Effect of essential oils from leaves of Ageratum conyzoides, Lantana camara and Chromolaena odorata on the mortality of Sitophilus zeamais (Coleoptera, Curculionidae). Journal of Stored Products Research, v.37, n.2, p.103-109, 2001.

CASTELLANI, D.C.; CASALI, V.W.D.; SOUZA, A.L.; CECON, P.R.; CARDOSO, C.A.; MARQUES, V.B. Produção de óleo essencial em canela (Ocotea odorifera Vell.) e guaçatonga (Casearia sylvestris Swartz) em função da época de colheita. Revista Brasileira de Plantas Medicinais, v.8, n.4, p.104-107, 2006.

CASTRO, H.G.; OLIVEIRA, L.O.; BARBOSA, L.C.A.; FERREIRA, F.A.; SILVA, D.J.H.; MOSQUIM, P.R.; NASCIMENTO, E.A. Teor e composição do óleo essencial de cinco acessos de mentrasto. Química Nova, v.27, n. 1, p.55-57, 2004.

CHANG, K.S.; AHN, Y.J. Fumigant activity of (E)-anethole identified in Illicum verum fruit against Blattella germanica. Pest Management Science, v.58, n.2, p.161-166, 2002.

COITINHO, R.L.B.C.; OLIVEIRA, J.V.; GONDIM JÚNIOR, M.G.C.; CÂMARA, C.A.G. Persistência de óleos essenciais em milho armazenado, submetido à infestação de gorgulho do milho. Ciência Rural, v.40, n.7, p.1492-14-96, 2010.

ESTRELA, J.L.V.; FAZOLIN, M.; CATANI, V.; ALÉCIO, M.R.; LIMA M.S. Toxicidade de óleos essenciais de Piper aduncum e Piper hispidinervum em Sitophilus zeamais. Pesquisa Agropecuária Brasileira, v.41, n.2, p.217-222, 2006.

FAZOLIN, M.; ESTRELA, J.L.V.; CATANI, V.; ALÉCIO, M.R.; LIMA, M.S. Propriedade inseticida dos óleos essenciais de Piper hispidinervum C.DC.; Piper aduncum L. e Tanaecium nocturnum (Barb. Rodr.) Bur. \& K.Shum sobre Tenebrio molitor L. Ciência e Agrotecnologia, Lavras, v.31, n.1 p. $113-120,2007$.
GUTIÉRREZ C.; FERERES, A.; REINA, M.; CABRERA, R.; GONZÁLESCOLOMA, A. Behavioral and sublethal effects of structurally related lower terpenes on Myzus persicae. Journal of Chemical Ecology, v.23, n.6, p.1641-1650, 1997.

HO, S.H.; MA, Y.; HUANG, Y. Anethole, a potential insecticide from Illicium verum Hook F., against two stored product insects. International Pest Control, v.39, p.50-51, 1997.

HUANG, Y.; HO, S.H.; KINI, R.M. Bioactivities of safrole and isosafrole on Sitophilus zeamais (Coleoptera: Curculionidae) and Tribolium castaneum (Coleoptera: Tenebrionidae). Journal of Economic Entomology, v.92, n.3, p.676-683, 1999.

HUANG, Y.; HO, S.H.; LEE, H.C.; YAP, Y.L. Insecticidal properties of eugenol, isoeugenol and methyleugenol and their effects on nutrition of Sitophilus zeamais Motsch. (Coleoptera: Curculionidae) and Tribolium castaneum (Herbst) (Coleoptera: Tenebrionidae). Journal of Stored Products Research, v.38, n.5, p.403-412, 2002.

KONG, C.; HU, F.; XU, T.; LU, Y. Allelopathic potential and chemical constituents of volatile oil from Ageratum conyzoides. Journal of Chemical Ecology, v.25, n.10, p.2347-2356, 1999.

Kosalec, I.; PEPELJNJAK, S.; KUSTRAK, D. Antifungal activity of fluid extract and essential oil from anise fruits (Pimpinella anisum L., Apiaceae). Acta Pharmaceutica, v.55, n.4, p.377-85, 2005.

LIMA, H.R.P.; KAPLAN, M.A.C.; CRUZ, A.M. Influência dos fatores abióticos na produção e variabilidade de terpenóides em plantas. Floresta e Ambiente, v.10, n.2, p.71-77, 2003.

LIMA, R.K.; CARDOSO, M.G.; MORAES, J.C.; MELO, B.A.; RODRIGUES, V.G.; GUIMARÃES, P.L. Atividade inseticida do óleo essencial de pimenta longa (Piper hispidinervum C. DC.) sobre lagarta-do-cartucho do milho Spodoptera frugiperda (J. E. Smith, 1797) (Lepidoptera: Noctuidae). Acta Amazônica, v.39, n.2, p. 377-38, 2009.

Lorenzi, H.; Matos, F.J.A. Plantas medicinais no Brasil: nativas e exóticas. Nova Odessa: Instituto Plantarum de Estudos da Flora, 2002, 512p.

MENDONÇA, F.A.C.; SILVA, K.F.; SANTOS, K.K.; RIBEIRO JÚNIOR, K.A.; SANT'ANA, A.E. Activities of some Brazilian plants against larvae of the mosquito Aedes aegypti. Fitoterapia, v.76, n.7-8, p.629-636, 2005.

NGOH, S.P.; CHOO, L.E.W.; PANG, F.Y.; HUANG, Y.; KINI, M.R.; HO, S.H. Insecticidal and repellent properties of nine volatile constituents of essential oils against the American cockroach, Periplaneta americana (L.). Pesticide Science, v.54, n.3, p.261-268, 1998.

OKUNADE, A.L. Ageratum conyzoides L. Asteraceae. Fitoterapia, v.73, n.1, p.1-16, 2002

PAVELA, R. Insecticidal activity of essential oils against cabbage aphid Brevicoryne brassicae. Journal of Essential Oil Bearing Plants, v.9, n.2, p.99-106, 2006. 
PINHEIRO, P.F.; QUEIROZ, V.T.; RONDELLI, V.M.; COSTA, A.V.; MARCELINO, T.P.; PRATISSOLI, D. Insecticidal activity of citronella grass essential oil on Frankliniella schultzei and Myzus persicae. Ciência e Agrotecnologia, v.37, n.2, p.138-144, 2013.

R DEVELOPMENT CORE TEAM. R: A language and environment for statistical computing. Vienna: R Foundation for Statistical Computing, 2010.

REGNAULT-ROGER, C. The potential of botanical essential oils for insect pest control. Integrated Pest Management Reviews, v.2, n. 1, p.25-34, 1997.

RIZZINI, C.T.; MORS, W.B. Botânica econômica brasileira. São Paulo: Editora Cultural, 1995. 241p.

RODRIGUES, V.M.; ROSA, P.T.; MARQUES, M.O.; PETENATE, A.J.; MEIRELES, M.A. A supercritical extraction of essential oil from aniseed (Pimpinella anisum L.) using CO2: solubility, kinetics, and composition data. Journal Agricultural and Food Chemistry, v.51, n.6, p. $1518-1523,2003$.

SAXENA, R.C.; DIXIT, O.P.; SUKUMARAN, P. Laboratory assessment indigenous plants extracts for anti-juvenile hormone activity in Culex quinquefasciatus. Indian Journal of Medical Research, v.95, p.204-206, 1992.
SIMÕES, C.M.; SPITZER, V. Óleos voláteis. In: SIMÕES, C.M.O.; SCHENKEL, E.P.; GOSMANN, G.; MELLO, J.C.P.; MENTZ, L.A.; PETROVICK, P.R. Farmacognosia: da planta ao medicamento. Porto Alegre: Editora da UFRGS, 2004. p.467-495.

SOARES, C.S.A.; SILVA, M.; COSTA, M.B.; BEZERRA, C.E.S.; CARVALHO, L.M.; SOARES, A.H.V. Atividade inseticida de óleos essenciais sobre Macrosiphum euphorbiae (Thomas) (Hemiptera: Aphididae) em roseira. Revista Brasileira de Agroecologia, v.7, n. 1, p.169-175, 2012.

SOARES, C.S.A.; COSTA, M. B.; SOARES, A.H.V.; BEZERRA, C.E.S.; CARVALHO, L.M. Avaliação da atividade inseticida do óleo essencial de mentrasto (Ageratum conyzoides L.) sobre o pulgão Macrosiphum euphorbiae (Thomas, 1878) (Hemiptera: Aphididae) em roseira. Revista Verde de Agroecologia e Desenvolvimento Sustentável, v.6, n.5, p.21-24, 2011.

VARDAR-UNLÜ, G.; CANDAN, F.; SÖKMEN, A.; DAFERERA, D.; POLISSIOU, M.; SÖKMEN, M.; DÖNMEZ, E.; TEPE, B. Antimicrobial and antioxidant activity of essential oil and methanol extract of Thymus pectinatus Fish. et Mey. var. Pectinatus (Lamiaceae). Journal of Agricultural and Food Chemistry, v.51, n.1, p.63-67, 2003. 